\title{
Assessment of Micro-vessel Density in Brain Gliomaby CD105 Expression
}

\author{
Hiva Saffar*, Marzieh Mirzaii, Elham Mirzaian, Farid Kosari
}

Dept. of Pathology, Shariati Hospital, Tehran University of Medical Sciences, Tehran, Iran

\begin{tabular}{c}
\hline KEYWORDS \\
\hline Glioma; \\
CD105; \\
Immunohistochemistry \\
\hline
\end{tabular}

Article Info

Received 29 Jan 2017; Accepted 05 April 2018; Published Online 17 July 2018;

\section{ABSTRACT}

Background \& Objective: Micro-vascular proliferation is an important histological feature of brain glioma with more vascular proliferation is present in higher grades of glioma. CD 105 is expressed in new actively proliferating and immature endothelial cells in tumor environment and appears to be capable to distinguish between malignant neo-vasculature and normal vessels.

Methods: This study was designed to evaluate the Micro-Vessel Density(MVD) in different grades of brain glioma based on CD 105 expression byImmunohistochemistry method to determine whether it can be a helpful marker for rumor grading or not.

Paraffin blocks of formalin fixed samples of brain astrocyticglioma were retrieved and IHC was performed using anti-CD105 monoclonal mouse antibody.

Results: Total number of 48cases of low and high grade astrocyticgliomas were evaluated. We noted that there was a positive correlation between MVD evaluated by CD105 and tumor grade, meaning that expression was significantly greater in tumors with higher grade $(P=0.019)$.

Conclusion: We concluded that MVD quantified by CD 105 has positive correlation with tumor grade. Also we think that expression of CD 105 specially in low-grade glioma can serve as a basis for selective treatment option in combination with current standard care.

Corresponding information:

Hiva Saffar, Associate Professor of Anatomical and Clinial Pathology, Dept. of Pathology, Shariati Hospital, Tehran University of Medical Sciences, Tehran, Iran. E-mail: Hsaffar@sina.tums.ac.ir

Copyright (C) 2018, IRANIAN JOURNAL OF PATHOLOGY. This is an open-access article distributed under the terms of the Creative Commons Attribution-noncommercial 4.0 International License which permits copy and redistribute the material just in noncommercial usages, provided the original work is properly cited.

\section{Introduction}

Gliomas are the most common primary brain tumors in adults $(1,2)$. Although molecular genetic features are included in recent World Health Organization (WHO) classification updates, its grade determinations are still based on histologic criteria (3).

Despite advances in treatment modalities, the prognosis of gliomas, especially higher grades, remains poor $(1,4)$.

The median survival is about six to eight years for low-grade (I, II) tumors, but decreases to two to five years for grade III and less than two years in grade IV (glioblastoma multiforme) $(1,4)$.

Only less than $3 \%$ of patients with glioblastoma multiforme survive more than five years (4).
Microvascular proliferation is an important histological feature of brain glioma (5) with more vascular proliferation present in higher grades of glioma. Glioblastoma is actually amongst the most vascularized tumors (6).

Histologic evaluation of tumor angiogenesis based on micro-vessel density (MVD) is an independent prognostic factor in patients diagnosed with glioma (5).

Some endothelial markers such as CD31, CD34, or Factor VIII are implicated, but they do not differentiate between mature vessels and microvasculature stimulated for tumor angiogenesis $(5,7,8)$.

CD105 was originally characterized more than two decades ago (2) and is a $180 \mathrm{kDa}$ integral membrane 
glycoprotein, which is an accessing receptor for the transforming growth factor beta $(2,6)$. It is specially expressed in new actively proliferating and immature endothelial cells in tumor environment $(2,5)$.

CD105 expression is implicated in diagnosis and prognosis assessment and as a treatment option in variable tumors including breast $(2,9)$, squamous cell carcinoma $(2,10)$, pancreatic ductal carcinoma $(2,11)$, non-small cell lung cancer and prostate cancer (6), and appears capable of distinguishing between malignant neo-vasculature and normal vessels (6).

Moreover, antibody based therapeutic strategies are considered as complementary treatment options in different neoplasms leading to notifying novel potential antigens (2).

Anti-angiogenic based target therapies with controversial results are undertaken in several clinical trials $(6,12,13)$

In this regard, it is suggested that CD105 antibody based treatment can be effective in preventing angiogenesis and inhibiting the formation of capillary-like structures with high specificity toward tumor tissue and less probable side effects (6).

On the other hand, recently, some studies denoted that the CD105 positive vascular structures play a clinical role in biology of gliomas with influence on tumor prognosis (2), but it is not clear whether CD105 can be used as complementary criteria for grading glioma (2).

The current study aimed at evaluating the MVD in different grades of glioma based on CD105 expression by immunohistochemistry (IHC) method to determine whether it can be a helpful marker for rumor grading or not.

Also, expression of CD105 in low-grade gliomas indicates a potential complementary therapeutic op- tion in lower grade tumors in order to prevent tumor recurrence.

\section{Materials and Methods}

Paraffin blocks of formalin-fixed samples of brain glioma from 2013 to 2014 were retrieved from the archive of Pathology Department, Shariati Hospital, Tehran, Iran. The slides were regraded according to WHO criteria (14); grade I, $n=8$; grade II, $n=16$; grade III, $n=8$; grade IV, $n=16$. The questionable cases were excluded.

IHC was performed using anti-CD105 monoclonal mouse antibody (4G11, Leica) and anti-CD31 mouse monoclonal antibody (JC/70A, Biogenex) according to the manufacturers' recommendations (CD31 study was performed to confirm localization of neo-vasculature endothelium).

The stained slides were evaluated for MVD based on CD105 staining $(4,15)$.

Briefly, the four most vascularized areas (hot spots) were selected at low power followed by counting each positive endothelial cell or cluster of endothelial cells ( \pm Lumina) at high magnification. The mean number of vessels in four areas was considered as density per high power field (HPF).

After data collection, the analysis of data was conducted with SPSS version 19. P-value $<0.05$ was considered significant.

\section{Results}

Total number of $8,16,8$, and 16 cases of grades I, II, III, and IV astrocytoma were evaluated, respectively.

Grades I and II tumors were categorized as low and grades III and IV as high.

The frequency of different grades regarding gender is summarized in Table 1.

Table 1. The Frequency of Different Grades Regarding Gender

\begin{tabular}{l|c|c|c|}
\hline \multirow{2}{*}{ Gemographic Data } & \multicolumn{2}{|c|}{ Gender } & \multirow{2}{*}{ Total } \\
\cline { 2 - 4 } LOW & Female & Male & 24 \\
High & 13 & 11 & 24 \\
\hline
\end{tabular}


207. Assessment of Micro-vessel Density ...

The mean age of patients with lower grade tumors was 26.7 years (ranged 10 to 35 ) in comparison with 46.6 years (ranged 22 to 71 ) in cases with high grades.

Regarding the vessel density, a positive correlation between MVD and tumor grades were observed, which meant that MVD was significantly greater in tumors with higher grades $(P=0.019)$. The data are summarized in Table 2.

Moreover, CD31 and CD105 revealed significant and positive correlation regarding the number of stained vessels $(P=0.012)$ (Figure 1$)$.

Table 2. Correlation Between MVD Evaluated by CD105 Expression and Tumor Grade

\begin{tabular}{ccccc}
\hline Grade & I & II & III & IV \\
\hline Mean MVD expression by CD105 & 8.37 & 8.31 & 19.87 & 25.25 \\
\hline
\end{tabular}
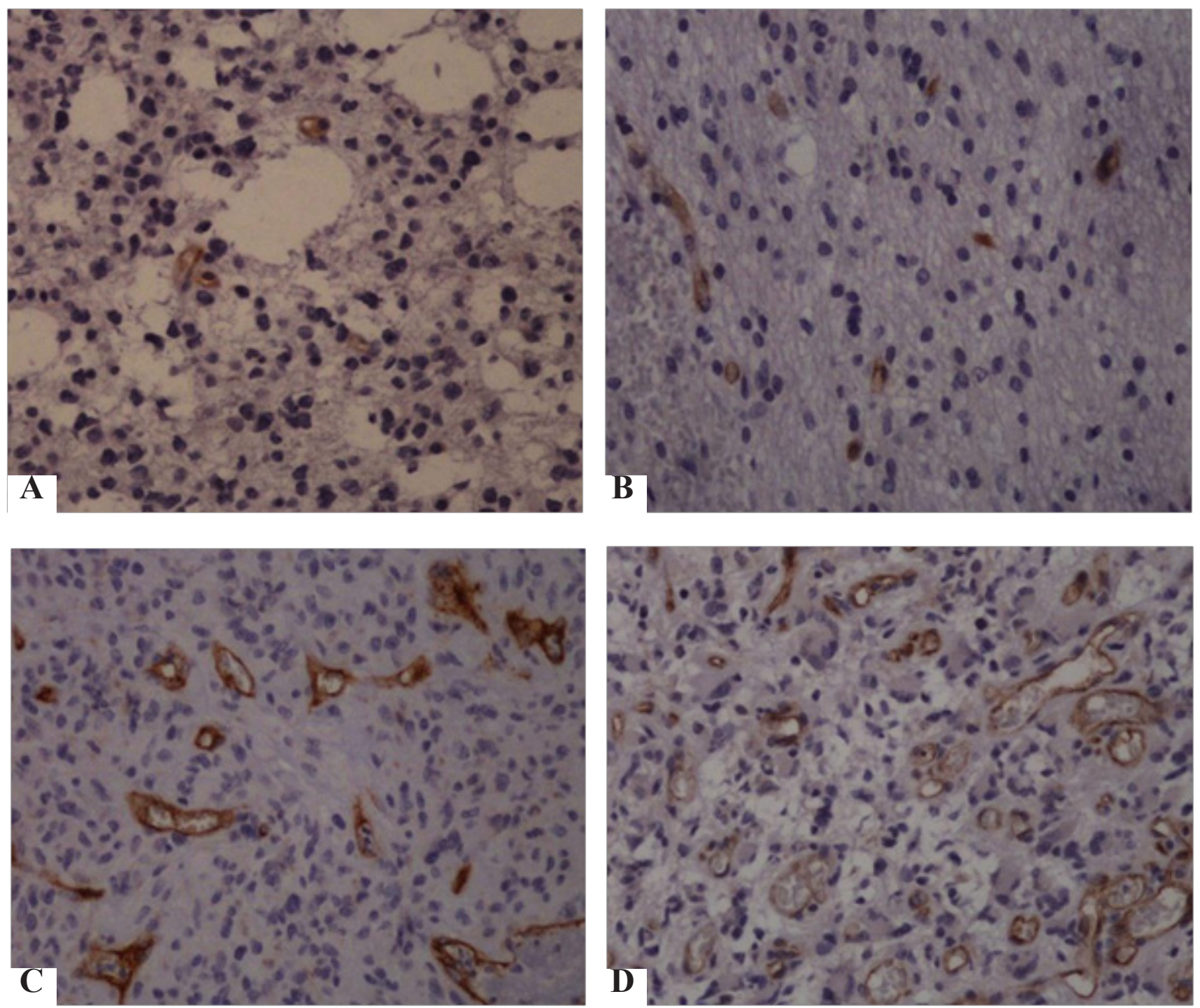

Figure 1. Expression of CD105 in vessels of gliomas with different grades (A) I; (B) II; (C) III; (D) IV 


\section{Discussion}

High-grade gliomas are highly vascular tumors (16). Angiogenesis is crucial to supply adequate nutrient and oxygenation for tumor growth and eliminate cellular waste product (16). Additionally, in combination with cellular proliferation and focal necrosis, microvascular proliferation is a key histologic feature in glioma grading (17).

It is suggested that intratumoral MVD quantified by IHC for endothelial cell markers correlate with prognosis, which meant that increase in MVD is associated with higher tumor grade (17) and shorter survival (18). Although the process of angiogenesis is subjected to many investigations, the role and function of endothelial cells still remain unclear (17). However, it is evident that tumor-associated vessels show altered shape and integrity (17). They lose blood brain barrier (BBB) properties with the increase in permeability (5), leading to fluid leakage into brain parenchyma and edema (17). Although tumor-associated vascularization was first introduced in 1940 (19-21), Dr. Judah Folkman in the early 1970s showed that targeting this neovascularization could be a vital strategy for cancer treatment $(20,22,23)$.

Among solid tumors, glioblastoma shows the most angiogenic feature with marked degree of vascular proliferation and endothelial cell hyperplasia (16), suggesting a potential novel therapeutic approach to target angiogenesis (16).

There are conflicting results whether or not the degree of angiogenesis measured by MVD is of prognostic value in astrocytic tumors (8). While most studies showed positive relationship between MVD and tumor survival, some others reported opposite findings (24). This partly could be due to variable antibodies used against different pan endothelial cell markers $(18,8)$, differences in counting method or staining procedures (18).

Efforts to standardize the method may be helpful; however, first of all, the optimal marker is an essential requirement (18).

The $C D 105$ is an integral membrane glycoprotein of transforming growth factor- $\beta$ receptor complex (25).
As a vascular marker, CD105 is preferentially expressed in immature vessels and some studies proposed it as a marker for tumor-associated angiogenesis $(5,16,8)$; while it is barely detectable in normal tissue (20).

The utility of this marker is investigated in various neoplasms such as glioblastoma, non-small cell lung cancer, and prostate cancer (6).

It is reported that CD105 MVD is increased with increase in tumor grade (5).

Jia et al., (5) reported that CD105 MVD was significantly lower in low-grade glioma in comparison with that of high-grade tumors, but there was no difference between grade III and IV tumors (5).

In the current study, the mean MVD in grades I to IV gliomas were 8.37, 8.31, 19.87 and 25.25, respectively, and it was noted that the density of vessels was significantly higher in high-grade tumors $(P=0.019)$.

The current study results were in concordant with those of Yao et al. (8). They evaluated 50 astrocytic tumors. The mean MVD in low-grade, as well as grade III and IV tumors were 24.8, 42.7, and 51.9, respectively. They found significant increase in MVD by $C D 105$ with increase in tumor grade. Moreover, patients with either glioblastoma or low-grade gliomas with higher MVD had significantly shorter mean survival time (8).

In another study, smith et al., (16) evaluated 150 grade III and IV pediatric glial tumors for MVD by CD105 and CD31. The mean MVD per core of tissue micro-array (TMA) was 8 and 6.4 for grade IV and III astrocytic tumors, respectively $(P=0.44)$.

They scored the density in a semi-quantitative fashion with less than 3/core TMA, 3-8, and $>8$ vessel/ TMA. All patients that survived for more than eight years had scores $<3 \mathrm{vessel} / \mathrm{core}$. Also, they found that $C D 105$ expression had a significant association with poor prognosis on multivariate analysis $(P<0.001)$ (16).

In a meta-analysis, Kong et al., (2) concluded that CD105 overexpression in glioma tissue was strongly linked to high grade of tumors. 
In the current study, $C D 31$ and $C D 105$ had positive correlation $(P=0.012)$. However, some studies $(8,24)$ concluded that CD105 was a better marker than CD31 to evaluate angiogenesis and prediction of prognosis in astrocytic tumors $(8,24)$.

Thus, measuring MVD by this marker is superior to traditional pan-endothelial markers such as $C D 1$, CD34, or Factor VIII (18).

Radiation is the most effective treatment for glioblastoma in combination with surgery, but the efficacy is limited due to radiation resistance (26); thus, some other therapeutic options are investigated.

Vascular endothelial growth factor $(V E G F)$, for example, is another potent growth factor mediating tumor angiogenesis and overexpression of VEGFR is associated with poor prognosis in glioblastoma (27). Bevacizumab is a recombinant humanized monoclonal antibody that binds to VEGF and suppresses $V E G F$ signaling, thereby downregulating angiogenesis $(27,28)$. Although it was initially observed that bevacizumab was associated with high radiographic response rate and prolonged progression-free survival, no overall survival benefit of bevacizumab was identified in multiple phase III trials (28).

Tumor specific expression of CD105 within vascular capillary beds was utilized in some therapeutic strategies $(20,29-33)$.

CD105 specific monoclonal antibodies can be fused to radioactive isotope or a toxic compound, which target tumor vasculature and inhibit tumor growth in pre-clinical mouse models of breast cancers $(20,29$, $31,33)$.

Zhong Zheng Jian et al., (5) demonstrated that expression of CD105 in peritumoral area was also of prognostic value in addition to those of the neoplastic tissue. Therefore, new therapeutic strategies are directed against tumor mass and can also target invasive cells in peritumoral normal appearing brain (6), which are probably beneficial to prevent tumor recurrence.

Finally, as most of other studies the current study also concluded that MVD quantified by CD105 had positive correlation with tumor grade. Also, regarding the potential utility of anti CD105 antibody for target therapy, the current study supposed that expression of CD105, especially in low-grade glioma, can serve as a basis for selective treatment option in combination with the current standard care in glioma. This approach can potentially improve treatment efficacy.

\section{Acknowledgment}

The authors would like to thank the staff of Pathology and Immunohistochemistry Department of Shariati Hospital for their technical support.

\section{Conflict of interests}

The authors declared no conflict of interest.

\section{References}

1. Guan Y, Chen L, Bao Y, Li Z, Cui R, Li G, et al. Identification of low miR-105 expression as a novel poor prognostic predictor for human glioma. Int J Clin Exp Med. 2015;8(7):10855-64. PMID:26379879 PMCID: PMC4565262

2. Kong X, Wang Y, Liu S, Xing B, Yang Y, Li Y, et al. CD105 over-expression is associated with higher WHO grades for gliomas. Mol Neurobiol. 2016;53(5):3503-12. https://doi.org/10.1007/ s12035-015-9677-1 PMID:26884265

3. Louis DN, Perry A, Reifenberger G, Von Deimling A, Figarella-Branger D, Cavenee WK, et al. The 2016 World Health Organization classification of tumors of the central nervous system: a summary. Acta Neuropathol. 2016;131(6):80320. https://doi.org/10.1007/s00401-016-1545-1 PMID:27157931

4. Moghaddam NA, Mahsuni P, Taheri D. Evaluation of endoglin as an angiogenesis marker in glioblastoma. Iran J Pathol. 2015;10(2):89-96. PMID: 26351468 PMCID:PMC4539765

5. Jia ZZ, Gu HM, Zhou XJ, Shi JL, Da Li M, Zhou GF, et al. The assessment of immature microvascular density in brain gliomas with dynamic contrast-enhanced magnetic resonance imaging. Eur J Radiol. 2015;84(9):1805-

9. https://doi.org/10.1016/j.ejrad.2015.05.035 PMID:26066470

6. Sica G, Lama G, Anile C, Geloso MC, La Torre G, De Bonis P, et al. Assessment of angiogenesis by CD105 and nestin expression in peritumor tissue of glioblastoma. Int J Oncol. 
2011;38(1):41-9. PMID:21109924

7. Sun H, Guo D, Su Y, Yu D, Wang Q, Wang T, et al. Hyperplasia of pericytes is one of the main characteristics of microvascular architecture in malignant glioma. PloS one. 2014;9(12):e114246. https://doi.org/10.1371/journal.pone.0114246 PMID:25478951 PMCID:PMC4257691

8. Yao Y, Kubota T, Takeuchi H, Sato K. Prognostic significance of microvessel density determined by an anti-CD105/endoglin monoclonal antibody in astrocytic tumors: Comparison with an anti-CD31 monoclonal antibody. Neuropathology. 2005;25(3):201-6. https://doi.org/10.1111/ j.1440-1789.2005.00632.x PMID:16193836

9. Martinez LM, Labovsky V, de Luján Calcagno M, Davies KM, Rivello HG, Bianchi MS, et al. CD105 expression on CD34-negative spindleshaped stromal cells of primary tumor is an unfavorable prognostic marker in early breast cancer patients. PloS one. 2015;10(3):e0121421. https://doi.org/10.1371/journal.pone.0121421 PMID:25803686 PMCID:PMC4372565

10. Nair S, Nayak R, Bhat K, Kotrashetti VS, Babji D. Immunohistochemical expression of CD105 and TGF- $\beta 1$ in oral squamous cell carcinoma and adjacent apparently normal oral mucosa and its correlation with clinicopathologic features. Appl Immunohistochem Mol Morphol. 2016;24(1):35-41. PMID:25710582

11. Lytras D, Leontara V, Kefala M, Foukas PG, Giannakou N, Pouliakis A, et al. Microvessel landscape assessment in pancreatic ductal adenocarcinoma: unclear value of targeting endoglin (CD105) as prognostic factor of clinical outcome. Pancreas. 2015;44(1):87-92. PMID:25058886

12. Friedman HS, Prados MD, Wen PY, Mikkelsen T, Schiff D, Abrey LE, et al. Bevacizumab alone and in combination with irinotecan in recurrent glioblastoma. J Clin Oncol. 2009;27(28):473340. https://doi.org/10.1200/JCO.2008.19.8721 PMID:19720927

13. Jain RK, di Tomaso E, Duda DG, Loeffler JS, Sorensen AG, Batchelor TT. Angiogenesis in brain tumors. Nat Rev Neurosci. 2007;8(8):610-22. https://doi.org/10.1038/nrn2175 PMID:17643088 Louis DN, Ohgaki H, Wiestler OD, Cavenee
WK.(eds). WHO classification of tumours of the Central Nervous System. Lyon: IARC; 2007.

15. Weidner N. Current pathologic methods for measuring intratumoral micrvessel density within breast carcinoma and other solid tumors. Breast Cancer Res Treat. 1995;36(2):169-80. PMID: $\underline{8534865}$

16. Smith SJ, Tilly H, Ward JH, Macarthur DC, Lowe J, Coyle B, et al. CD105 (Endoglin) exerts prognostic effects via its role in the microvascular niche of paediatric high grade glioma. Acta Neuropathol. 2012;124(1):99-110. https://dx.doi.org/10.1007\%2Fs00401-012-0952-1 PMID:22311740 PMCID:PMC3377898

17. Miebach S, Grau S, Hummel V, Rieckmann P, Tonn J-C, Goldbrunner RH. Isolation and culture of microvascular endothelial cells from gliomas of different WHO grades. J Neurooncol. 2006;76(1):39-48. https://doi.org/10.1007/s11060005-3674-6 PMID: $\underline{16155723}$

18. 18. Nassiri F, Cusimano MD, Scheithauer BW, Rotondo F, Fazio A, Yousef GM, et al. Endoglin (CD105): a review of its role in angiogenesis and tumor diagnosis, progression and therapy. Anticancer Res. 2011;31(6):2283-90. PMID:21737653

19. Algire GH, Chalkley HW, Legallais FY, Park HD. Vascular reactions of normal and malignant tissues in vivo. I. Vascular reactions of mice to wounds and to normal and neoplastic transplants. J Natl Cancer Inst. 1945;6(1):7385. https://doi.org/10.1093/jnci/6.1.73

20. Wood LM, Pan Z-K, Guirnalda P, Tsai P, Seavey M, Paterson Y. Targeting tumor vasculature with novel Listeria-based vaccines directed against CD105. Cancer Immunol Immunother. 2011;60(7):931-42. https://doi. org/10.1007/s00262-011-1002-x PMID:21431419 PMCID:PMC4438988

21. Younger J, Algire G. The effect of vascular occlusion on transplanted tumors. J Natl Cancer Inst. 1949;10(3):565-79.

22. Folkman J. Tumor angiogenesis: therapeutic implications. N Engl J Med. 1971;285(21):11826. https://doi.org/10.1056/NEJM197111182852108 PMID:4938153 
23. Folkman J, Merler E, Abernathy C, Williams $\mathrm{G}$. Isoltion of a tumor factor responsible for angiogenesis. J Exp Med. 1971;133(2):275-88. PMID:4332371 PMCID:PMC2138906

24. Behrem S, Zarkovic K, Eskinja N, Jonjic N. Endoglin is a better marker than CD31 in evaluation of angiogenesis in glioblastoma. Croat Med J. 2005;46(3):417-22. PMID: 15861521

25. Luque A, Slevin M, Turu MM, Juan-Babot O, Badimon L, Krupinski J. CD105 positive neovessels are prevalent in early stage carotid lesions, and correlate with the grade in more advanced carotid and coronary plaques. J Angiogenes Res. 2009;1(1):6. https://dx.doi. org/10.1186\%2F2040-2384-1-6 PMID: 19946414 PMCID:PMC2776238

26. Hovinga KE, Shimizu F, Wang R, Panagiotakos G, Van Der Heijden M, Moayedpardazi H, et al. Inhibition of notch signaling in glioblastoma targets cancer stem cells via an endothelial cell intermediate. Stem Cells. 2010;28(6):1019-29. https://doi.org/10.1002/stem.429 PMID:20506127 PMCID:PMC5532884

27. Abdelmaksoud BA. Updates in Genetic Molecular Targeted Therapy for Glioblastoma. Cancer and Oncology Research. 2016;4(1):1-

15. http://www.hrpub.org/journals/article info. php?aid $=3424$

28. Chen R, Cohen AL, Colman H. Targeted therapeutics in patients with high-grade gliomas: past, present, and future. Curr Treat Options
Oncol. 2016;17(8):42. https://doi.org/10.1007/ s11864-016-0418-0 PMID:27334978

29. Benítez J, Ferreras JM, Muñoz R, Arias Y, Iglesias R, Córdoba-Díaz M, et al. Cytotoxicity of an ebulin 1-anti-human CD105 immunotoxin on mouse fibroblasts (L929) and rat myoblasts (L6E9) cells expressing human CD105. Med Chem. 2005;1(1):65-71. PMID:16789887

30. Lee S-H, Mizutani N, Mizutani M, Luo Y, Zhou H, Kaplan C, et al. Endoglin (CD105) is a target for an oral DNA vaccine against breast cancer. Cancer Immunol Immunother. 2006;55(12):1565-74. https://doi.org/10.1007/ $\underline{\mathrm{s} 00262-006-0155-5}$ PMID: $\underline{16565828}$

31. Matsuno F, Haruta Y, Kondo M, Tsai H, Barcos $\mathrm{M}$, Seon BK. Induction of lasting complete regression of preformed distinct solid tumors by targeting the tumor vasculature using two new anti-endoglin monoclonal antibodies. Clin Cancer Res. 1999;5(2):371-82. PMID: 10037187

32. Mizutani N, Luo Y, Mizutani M, Reisfeld R, xiang R. DNA vaccines suppress angiogenesis and protect against growth of breast cancer metastases. Breast Dis. 2004;20:81-91. PMID: $\underline{15687710}$

33. Tabata M, Kondo M, Haruta Y, Seon BK. Antiangiogenic radioimmunotherapy of human solid tumors in SCID mice using (125)I-labeled anti-ndoglin monoclonal antibodies. Int J Cancer. 1999;82(5):737-42. PMID: 10417773

How to Cite This Article

Saffar H, Mirzaii M, Mirzaian E, Kosari F. Assessment of Micro-vessel Density in Brain Glioma by CD105 Expression. Iranian Journal of Pathology, 2018; 13(2): 205-211. 